\title{
Analytical Design of Double-Chirped Mirrors with Custom-Tailored Dispersion Characteristics
}

\author{
Nicolai Matuschek, Franz X. Kärtner, and Ursula Keller, Member, IEEE
}

\begin{abstract}
We present a theory for the analytical design of double-chirped mirrors with special dispersion characteristics. A simple analytical equation takes an arbitrarily desired group delay dispersion (that also includes possible higher order dispersion) as an input function and gives the chirp law as an output. The chirp law determines the local Bragg wavelengths in the mirror. It allows the calculation of the thicknesses of the highand low-index layers if the double chirp of the layers in the front part of the mirror is taken into account. We use this method to design a highly dispersive double-chirped semiconductor Bragg mirror and a double-chirped $\mathrm{TiO}_{2}-\mathrm{SiO}_{2}$ mirror for higher order dispersion compensation in optical parametric oscillators operating in the visible spectral range. The design formulas are applicable to general chirped Bragg gratings and provide insight into the reasons why certain dispersion characteristics might be impossible to achieve.
\end{abstract}

Index Terms - Chirped mirrors, coatings, coupled-mode analysis, dielectric films, dispersion control, thin-film devices, ultrafast optics, WKB analysis.

\section{INTRODUCTION}

$\mathbf{R}$ APID advances in ultrashort pulse generation have resulted in sub-10-fs pulses from Ti:sapphire lasers [1]-[4]. It became evident that the main limitation to short pulse generation is given by higher order dispersion. Chirped mirrors [5] provide a powerful and compact technique for dispersion compensation. Additionally, they exhibit a broader highreflectance range than standard quarter-wave Bragg mirrors. A chirped mirror introduces a controlled negative group delay dispersion (GDD) by increasing the local Bragg period of the mirror along the grating.

However, so far there were no analytical laws for the chirping of the layer thicknesses for mirrors with a given dispersion characteristic. Therefore, the mirrors are often designed by time-consuming numerical optimization. The reason for this lack in appropriate design formulas is that the standard design methods for optical interference coatings were mostly focused on their reflection and transmission properties but not on the phase properties of the coatings. There exist only a few papers concerning the phase properties of multilayer coatings by analytical means (see, for example, [6]). Most of these results are only of a qualitative nature and give little analytical insight into the design problem. Additionally, they

Manuscript received June 5, 1998; revised October 20, 1998. This work was supported by the Swiss National Science Foundation.

The authors are with the Swiss Federal Institute of Technology (ETH), Institute of Quantum Electronics, Ultrafast Laser Physics Laboratory, ETH Hönggerberg-HPT E9, CH-8093 Zurich, Switzerland.

Publisher Item Identifier S 0018-9197(99)00857-X. do not answer important questions such as "Is it possible to design chirped mirrors with some arbitrarily chosen dispersion characteristics?" or "What is a good starting structure for a chirped mirror that can be used for computer optimization, given the desired reflectance and GDD of the mirror?"

Recently, we developed a theory for chirped mirror analysis that resulted in the double-chirped mirror (DCM) design technique [7], [8]. The theory, which is based on an exact coupled-mode analysis [9], allows for an easy and still precise calculation of the group delay and GDD by using the WKB approximation. In the case of weakly index-modulated nonuniform grating structures (e.g., chirped fiber gratings), the usefulness of the WKB approach has been already demonstrated by Poladian and Sipe et al. [10], [11]. In this paper, we use our analytical expressions and derive the chirp law for the design of mirrors with an arbitrary dispersion characteristic.

This paper widely uses the notation and results derived in [8] and is organized as follows. In Section II, the main results of [8] are summarized in a self-contained manner. In Section III, a differential equation for the chirp law, given a desired GDD, is derived within certain approximations. Subsequently, in Section IV, we use the analytical solution of this differential equation and explicitly determine the chirp law for some analytically and some numerically solvable cases. Finally, in Section V, the applicability and use of the derived formulas is demonstrated by investigation of two examples, a semiconductor Bragg mirror with a constant negative GDD and a dielectric mirror for dispersion compensation in optical parametric oscillators (OPO's). We discuss the final design of the dispersion compensating OPO mirrors obtained from the theoretical starting design after numerical refinement. These DCM's have the potential to support 6-fs pulses from an OPO in the visible [12].

\section{WKB DESCRIPTION OF A DOUBle-ChIRPED MiRRor}

According to Fig. 1, a DCM is an optical interference coating that in general consists of four multilayer subsections deposited on a substrate. For dispersion-compensating broadband high reflectors, the layer materials are dielectrics such as $\mathrm{SiO}_{2}$ and $\mathrm{TiO}_{2}$. The first section of a DCM is a broadband antireflection (AR) coating, which typically consists of 8-14 layers and matches the subsequent optical coating to the ambient medium [7], [8]. Here, we do not consider the problem of finding a proper high-quality AR coating (see, for example, [13]). We start to design the phase and amplitude properties of the mirror under the assumption of perfect matching to the ambient medium. Therefore, we count the layers starting 


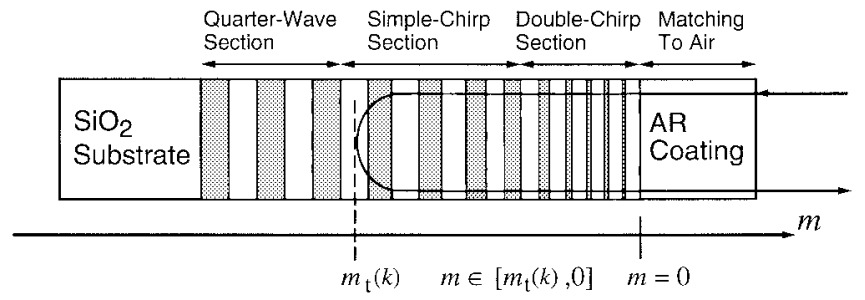

Fig. 1. Schematic drawing of a DCM, which is composed of four multilayer sections. The different task of each section is described in the text. The position in the mirror is determined by the variable $m$, where $m=0$ defines the beginning of the theoretical DCM structure directly after the AR coating. Light, with wavenumber $k$, is reflected at the wavenumber-dependent classical turning point $m_{\mathrm{t}}(k)$

from the first layer following the AR-coating section and we calculate all quantities with respect to this layer, which we assume to be made of the low-index material.

The double-chirp section is necessary for the avoidance of Gires-Tournois-like oscillations in the group delay. Double chirping means that the local Bragg wavelength of the index grating and the local coupling of the incident wave to the reflected wave are chirped simultaneously. This corresponds to an adiabatic matching of the impedance of the Bragg grating to the low-index layer at the front, as discussed in [7], [8]. In the subsequent simple-chirp section (Fig. 1), only the Bragg wavelength is chirped. This means that the highand low-index layers are quarter-wave layers for maximum reflection of waves with a wavelength close to the local Bragg wavelength. The fourth section is a quarter-wave Bragg mirror with fixed Bragg wavelength. The third and fourth sections are optional and depend on the special design problem considered. However, they are necessary to obtain high reflectances over a wavelength range as broad as possible.

The following theoretical derivation of the chirp law, as presented in Sections III and IV, deals only with the doublechirp and simple-chirp sections of the DCM and does not include the quarter-wave section. The reason is that wavelengths around the fixed Bragg wavelength of the quarter-wave section are reflected approximately at the same position in the mirror. This leads to an almost vanishing dispersion and the one-to-one mapping of the wavelength with its point of reflection (classical turning point) is lost. However, the quarter-wave stack at the end of the mirror can be considered as a continuation of the simple-chirp section in the case of a desired small amount of negative dispersion for the long wavelengths, as will be explained in more detail in Section V-C.

The Bragg grating is decomposed into symmetrically defined index steps [7], [8], and the discrete variable $(-m) \in \mathbb{N}$ counts the index steps of the chirped Bragg grating (see Fig. 2). In [7] and [8], we found an exact description of a chirped Bragg grating by exact coupled-mode equations of the form

$$
\frac{d}{d m}\left(\begin{array}{l}
A(m) \\
B(m)
\end{array}\right)=i\left(\begin{array}{cc}
-\delta(m) & -\kappa(m) \\
\kappa(m) & \delta(m)
\end{array}\right)\left(\begin{array}{l}
A(m) \\
B(m)
\end{array}\right)
$$

where $A$ and $B$ are the slowly varying amplitudes of the forward and backward propagating waves, respectively. Here, $m$ is considered to be a continuous variable, where the

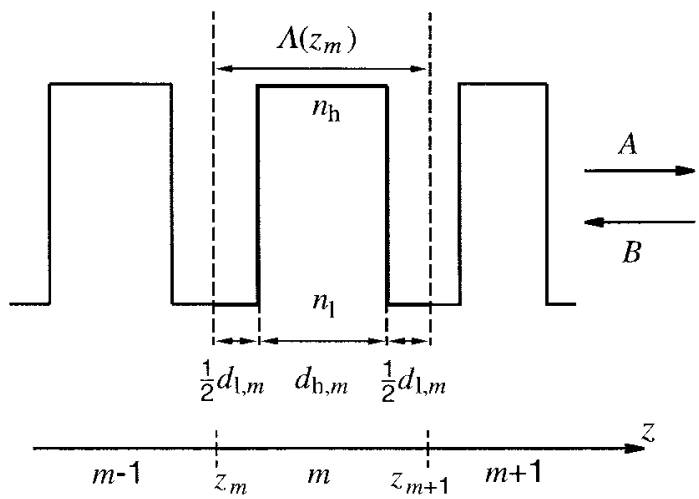

Fig. 2. Refractive-index profile of a DCM composed of two different layer materials with the indices $n_{\mathrm{h}}$ and $n_{1}$. The physical thickness of the symmetrically defined index steps is given by $\Lambda\left(z_{m}\right)=d_{\mathrm{h}, m}+d_{1, m}$, where $d_{\mathrm{h} / 1, m}$ denotes the physical thickness of the high-/low-index layer at the $(-m)$ th index step.

connection to the $z$ coordinate is given via $d m=d z / \Lambda\left(z_{m}\right)$, where $\Lambda\left(z_{m}\right)=d_{\mathbf{h}, m}+d_{1, m}$ is the physical thickness of the individual index step at position $z_{m}$. Thus, in (1), the thickness of a complete index step is normalized to $\Delta m=1$. Note that we place the mirror on the negative side of the $m$ axis, i.e., $m \leq 0$, where $m=0$ defines the beginning of the mirror (see Fig. 1). As we have recently shown, a chirped mirror is exactly described by the coupled-mode equations (1) even for arbitrarily high index differences of the layer materials, if the exact normalized coupling coefficient $\kappa$ and detuning coefficient $\delta$ are used [8], [9].

In [8], we used the WKB approximation to derive the following expression for the phase $\phi_{\mathrm{r}}$ of the wave reflected by a DCM with an ideally matched front part of the mirror, which is achieved by the double-chirp section:

$$
\phi_{\mathrm{r}}(k)=\frac{\pi}{2}+2 \cdot \int_{m_{\mathrm{t}}}^{0} q(m) d m, \quad m \in\left[m_{\mathrm{t}}, 0\right]
$$

with

$$
q(m)=\sqrt{-U_{0}}=\sqrt{\delta^{2}(m)-\kappa^{2}(m)}
$$

Here, $k$ is the vacuum wavenumber. The propagation constant $q$ in the grating is the square root of the negative scattering potential $U_{0}$ of the Schrödinger equation, which is equivalent to the coupled-mode equations (1), and $m_{\mathrm{t}}$ is the right turning point of the corresponding classical motion. The classical turning points are defined by the condition

$$
q\left(m_{\mathrm{t}}\right) \stackrel{!}{=} 0 \Rightarrow \delta\left(m_{\mathrm{t}}\right)= \pm \kappa\left(m_{\mathrm{t}}\right)
$$

In the following, we consider only generic potentials with one or two classical turning points [8, Fig. 6], where the right turning point corresponds to the positive sign in (4). In Fig. 3 , the negative potential $-U_{0}$ and the effective wavenumber $q$ are shown for a typical DCM for a wavelength in the highreflectance region. The area $\boldsymbol{A}$ in the figure corresponds to the integral that has to be calculated when evaluating (2). 


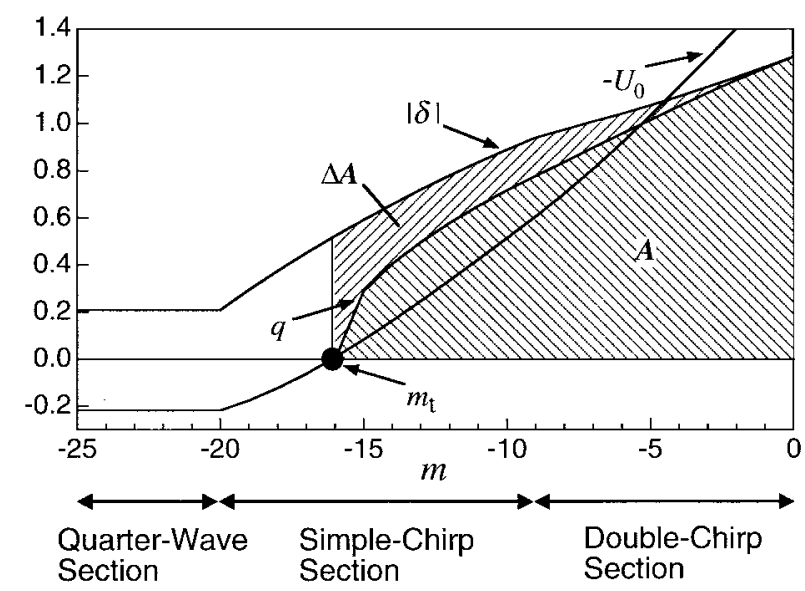

Fig. 3. The effective propagation constant $q(m)$ in the grating is the square root of the negative scattering potential $-U_{0}(m)$. The phase of the reflected light is essentially given by twice the area $A$ under the $q(m)$-curve, according to (2). The area has to be integrated from the beginning of the mirror $(m=0)$ to the right classical turning point $m_{\mathrm{t}}$. In the zeroth-order approximation, one has to integrate over the absolute value of the detuning coefficient $|\delta(m)|$. The error in the phase due to this approximation is indicated by the additional area $\Delta \boldsymbol{A}$. The curves in this figure are obtained for a typical wavelength in the high-reflectance range of a dielectric DCM.

\section{Analytical Derivation of the Chirp LAW}

In this section, we derive approximate expressions for the group delay and the GDD, based on (2). The relation for the GDD can also be interpreted as a differential equation for the classical turning point of the Bragg grating as a function of wavenumber, if a certain GDD is desired.

\section{A. Approximation of the WKB Formulas}

Generally, it is not possible to derive analytical expressions for the GDD due to the complicated integrand $q(m)=$ $\sqrt{\delta^{2}-\kappa^{2}}$ in (2). Therefore, we expand the square root in a Taylor series according to

$$
\sqrt{\delta^{2}(m)-\kappa^{2}(m)}=|\delta| \cdot \sum_{\eta=0}^{\infty}\left(\begin{array}{c}
1 / 2 \\
\eta
\end{array}\right)\left(-\frac{\kappa^{2}}{\delta^{2}}\right)^{\eta}
$$

and take only the zeroth-order term $|\delta|$ for an analytical estimation of the phase properties. The expansion (5) is always possible since $\kappa^{2} \leq \delta^{2}$ in the interval $\left[m_{\mathrm{t}}, 0\right]$. Fig. 3 also shows the error $\Delta \boldsymbol{A}$ in the integral (2) in zeroth-order approximation. Note that, in the impedance-matched case considered here, the propagation constant $q$ and its approximation $|\delta|$ have the same tangent at the beginning of the mirror, i.e., $m=0$.

In principle, one has to use the exact coupling and detuning coefficients for the calculation of the phase integral. However, for an analytical treatment of the DCM dispersion, further approximations are necessary. Therefore, in this paper, we use the expressions

$$
\begin{aligned}
& \kappa(m)=\kappa_{0}=-2 r=\text { const. } \\
& \delta(m)=\pi \cdot\left(\frac{k}{k_{\mathrm{B}}(m)}-1\right)
\end{aligned}
$$

which agree with the coefficients from standard coupled-mode theory [14]. Here, we have introduced the Fresnel reflectivity

$$
r=\frac{n_{\mathbf{h}}-n_{1}}{n_{\mathbf{h}}+n_{1}}
$$

where $n_{\mathbf{l}}$ and $n_{1}$ are the refractive indices of the high- and low-index layer materials, respectively. In the following, we denote

$$
k_{\mathrm{B}}(m)=\frac{2 \pi}{\lambda_{\mathrm{B}}(m)}=\frac{\pi}{n_{\mathbf{h}} d_{\mathbf{h}, m}+n_{1} d_{1, m}}
$$

as the local Bragg wavenumber and $\lambda_{\mathrm{B}}=2 \cdot\left(n_{\mathrm{h}} d_{\mathrm{h}, m}+\right.$ $\left.n_{1} d_{1, m}\right)$ as the corresponding local Bragg wavelength, where $d_{\mathbf{h}, m}$ and $d_{1, m}$ are the physical layer thicknesses of the highand low-index layers, respectively, at the $(-m)$ th index step (see Fig. 2).

\section{B. Chirp Laws for the Bragg Wavenumber}

Using the approximations (5)-(7), we derive the group delay from (2)

$$
\begin{aligned}
T_{\mathrm{g}}(k) & =-\frac{2}{c} \frac{\partial}{\partial k} \phi_{\mathrm{r}} \\
& =-\frac{2}{c} \int_{m_{\mathrm{t}}(k)}^{0}\left(\frac{\partial}{\partial k}|\delta(m)|\right) d m \\
& =\frac{2 \pi}{c} \int_{m_{\mathrm{t}}(k)}^{0} \frac{1}{k_{\mathrm{B}}(m)} d m \\
& =\frac{1}{c} \int_{m_{\mathrm{t}}(k)}^{0} \lambda_{\mathrm{B}}(m) d m
\end{aligned}
$$

where $c=\omega / k$ is the velocity of light in vacuum with the angular frequency $\omega$. Note that the second equality holds because the integrand (3) vanishes at the classical turning point according to (4). Thus, we first built the derivative of (2) with respect to wavenumber, and afterwards we applied the zerothorder approximation (5). The approximate group delay (10) has a simple physical interpretation. It is the optical path length for a wave traveling from the mirror surface to the classical turning point and back. From (10), we obtain the GDD

$$
\begin{aligned}
G D D(k) & =\frac{1}{c} \frac{\partial}{\partial k} T_{\mathrm{g}}(k) \\
& =-\frac{2 \pi}{c^{2} k_{\mathrm{B}}\left(m_{\mathrm{t}}(k)\right)} \frac{\partial}{\partial k} m_{\mathrm{t}}(k) .
\end{aligned}
$$

The meaning of this equation is twofold. The equation allows one to calculate the dispersion if the Bragg wavenumber is given as a function of the index step $k_{\mathrm{B}}(m)$, which also specifies the classical turning point as a function of wavenumber. The equation can also be interpreted as a firstorder differential equation for the classical turning point if a certain dispersion $G D D(k)$ is desired. Here, we are interested in the second interpretation, since it gives us the Bragg wavenumber for the index steps, and therefore the layer thicknesses, for a desired dispersion characteristic. Thus, we write

$$
\frac{\partial}{\partial k} m_{\mathrm{t}}(k)=-\frac{c^{2}}{2\left(\pi-\left|\kappa_{0}\right|\right)} k \cdot G D D(k)
$$


where we have used the equation for the classical turning point

$$
k_{\mathrm{B}}\left(m_{\mathrm{t}}(k)\right)=\frac{1}{1-\frac{\left|\kappa_{0}\right|}{\pi}} k \Leftrightarrow k\left(k_{\mathrm{B}}\right)=\left(1-\frac{\left|\kappa_{0}\right|}{\pi}\right) \cdot k_{\mathrm{B}}
$$

which follows from condition (4) with the approximations (6) and (7). Equation (13) shows that, for negligible coupling coefficients, the incident wave is reflected at the point where the wavenumber equals the Bragg wavenumber. This happens where the approximate detuning coefficient vanishes according to $(7)$. by

The general solution of the differential equation (12) is given

$$
m_{\mathrm{t}}(k)=a-\frac{c^{2}}{2\left(\pi-\left|\kappa_{0}\right|\right)} \cdot \int^{k} \bar{k} \cdot G D D(\bar{k}) d \bar{k}
$$

where $a$ is an arbitrary integration constant. This equation determines the classical turning point for a given wavenumber $k$. By using (13), which connects the Bragg wavenumber $k_{\mathrm{B}}$ at the classical turning point with the wavenumber $k$, the classical turning point can be written as a function of the corresponding Bragg wavenumber. Thus, we get the following inverse law for chirping the Bragg wavenumber with position $m$ in the mirror:

$$
m\left(k_{\mathrm{B}}\right)=a-\frac{c^{2}}{2 \pi}\left(1-\frac{\left|k_{0}\right|}{\pi}\right) \cdot \int^{k_{\mathrm{B}}} \bar{k}_{\mathrm{B}} \cdot G D D\left(\bar{k}_{\mathrm{B}}\right) d \bar{k}_{\mathrm{B}} .
$$

Note that the index $t$ at the variable $m$ is no longer necessary, since we consider $m$ as an independent variable. The integration constant is determined by a boundary condition-for example, the Bragg wavenumber at the beginning of the mirror. Hence, we have a condition of the form

$$
m\left(k_{\mathrm{B}}^{\max }\right) \stackrel{!}{=} 0
$$

where $k_{\mathrm{B}}^{\max }$ denotes the maximum Bragg wavenumber of a chirped mirror that produces a negative GDD. In that case, the Bragg wavenumber is a monotonically decreasing function with respect to the negative $m$ axis.

Using (16), we obtain the following closed-form solution for the turning point:

$$
m\left(k_{\mathrm{B}}\right)=\frac{c^{2}}{2 \pi}\left(1-\frac{\left|\kappa_{0}\right|}{\pi}\right) \cdot \int_{k_{B}}^{k_{\mathrm{B}}^{\max }} \bar{k}_{\mathrm{B}} \cdot G D D\left(\bar{k}_{\mathrm{B}}\right) d \bar{k}_{\mathrm{B}} .
$$

This equation allows us to calculate the position of the index step at which the Bragg wavenumber has the value $k_{\mathrm{B}}$ in order to achieve the given GDD. The inverse of this equation determines the Bragg wavenumber and, therefore, according to (9), the optical thickness as a function of the index step. At first, (17) might look surprising, since it contains the product $k_{\mathrm{B}} \cdot G D D\left(k_{\mathrm{B}}\right)$ and not only $G D D\left(k_{\mathrm{B}}\right)$, because that would relate to the change of the group delay over a distance of the index steps, if each step would have equal optical length. But this is not the case, and the optical thickness of the $|m|$ th step is proportional to the inverse of $k_{\mathrm{B}}$.

\section{ANALyTiCAL EXAMPLES}

In this section, we analytically discuss examples for using (17).

\section{A. Analytically Invertible Examples}

For some simple cases of the desired GDD, the chirp law can be derived explicitly. This is the case for a GDD that obeys a power law of the form

$$
G D D(k)=-D_{0} \cdot\left(\frac{k}{k_{0}}\right)^{\gamma}, \quad D_{0}>0, \gamma \in \mathbb{R} .
$$

Here, $D_{0}$ is the absolute value of the desired GDD at wavenumber $k_{0}$. For such a GDD, the third-order dispersion (TOD) is given by

$$
\operatorname{TOD}(k)=-\frac{\gamma D_{0}}{c k_{0}} \cdot\left(\frac{k}{k_{0}}\right)^{\gamma-1} .
$$

One can see that the TOD is positive for $\gamma<0$, negative for $\gamma>0$, and vanishes for $\gamma=0$. If we substitute (13) into (18), the calculation of the integral in (17) results in

$$
\begin{aligned}
m\left(k_{\mathrm{B}}\right) & =-\frac{c^{2}}{2 \pi}\left(1-\frac{\left|\kappa_{0}\right|}{\pi}\right)^{\gamma+1} \frac{D_{0}}{k_{0}^{\gamma}} \cdot \int_{\kappa_{\mathrm{B}}}^{k_{\mathrm{B}}^{\max }} \bar{k}_{\mathrm{B}}^{\gamma+1} d \bar{k}_{\mathrm{B}} \\
& =\left\{\begin{array}{cc}
-\frac{c^{2} D_{0}}{2 \pi(\gamma+2) k_{0}^{\gamma}}\left(1-\frac{\left|\kappa_{0}\right|}{\pi}\right)^{\gamma+1} & \gamma \neq-2 \\
\cdot\left(\left(k_{\mathrm{B}}^{\max }\right)^{\gamma+2}-k_{\mathrm{B}}^{\gamma+2}\right), & \gamma \neq-2 . \\
-\frac{c^{2} k_{0}^{2} D_{0}}{2\left(\pi-\left|\kappa_{0}\right|\right)} \cdot \ln \left(\frac{k_{\mathrm{B}}^{\max }}{k_{\mathrm{B}}}\right), & \gamma=-2 .
\end{array}\right.
\end{aligned}
$$

The inverse relationship leads to (21), shown at the bottom of the page, where we have used $m=-|m|$. Note that the chirp law for the case $\gamma=-2$ follows directly from the case $\gamma \neq-2$ in the limit $\gamma \rightarrow-2$. As an example, to obtain a constant negative GDD $(\gamma=0)$, one has to chirp the Bragg wavenumber according to the following square root law:

$$
k_{\mathrm{B}}(m)=k_{\mathrm{B}}^{\max } \cdot \sqrt{1-\frac{4 \pi^{2}}{c^{2} D_{0}\left(\pi-\left|\kappa_{0}\right|\right)\left(k_{\mathrm{B}}^{\max }\right)^{2}}|m|}
$$

$$
k_{\mathrm{B}}(m)= \begin{cases}k_{\mathrm{B}}^{\max } \cdot\left(1-\frac{2 \pi^{\gamma+2}(\gamma+2) k_{0}^{\gamma}}{c^{2} D_{0}\left(\pi-\left|\kappa_{0}\right|\right)^{\gamma+1}\left(k_{\mathrm{B}}^{\max }\right)^{\gamma+2}}|m|\right)^{1 /(\gamma+2)}, & \gamma \neq-2 \\ k_{\mathrm{B}}^{\max } \cdot \exp \left(-\frac{2\left(\pi-\left|\kappa_{0}\right|\right)}{c^{2} D_{0} k_{0}^{2}}|m|\right), & \gamma=-2\end{cases}
$$


which is a rather unexpected result. An example using this analytical chirp law will be discussed in detail in Section VA in the context of the design of a chirped semiconductor mirror. Reference [15] shows a comparison of group delays with different TOD's obtained when using (21) for different values of the chirp exponent $\gamma$.

Since we will want to compare the exact calculated group delay with the group delay obtained from the WKB solution, we give the group delay for the chirp law (21) according to (10) by using (21) and (13) for the evaluation of the integral and determination of the classical turning point. The result, (23), is shown at the bottom of the page. In the same way as mentioned above, the case $\gamma=-1$ follows from the $\gamma \neq-1$ case in the limit $\gamma \rightarrow-1$.

\section{B. Taylor Expansion of the Desired Dispersion Characteristic}

In general, the relationship (17) cannot be inverted. However, we can always compute the index step $(-m)$ as a function of Bragg wavelength if the dispersion is given as a Taylor expansion around a center wavenumber $k_{0}$

$$
\begin{aligned}
G D D(k) & =\sum_{v=0}^{\infty} \frac{1}{v !} D_{(v+2)} c^{v}\left(k-k_{0}\right)^{v} \\
& =\sum_{v=0}^{\infty} \sum_{\mu=0}^{v} \frac{1}{v !}\left(\begin{array}{l}
v \\
\mu
\end{array}\right) D_{(v+2)} c^{v}\left(-k_{0}\right)^{v-\mu} k^{\mu} .
\end{aligned}
$$

Here, we have introduced the dispersion coefficients

$$
D_{(v+2)}=\left.\frac{1}{c^{v}} \frac{\partial^{v}}{\partial k^{v}} G D D(k)\right|_{k=k_{0}}, \quad v \in \mathbb{N}_{0}
$$

Substitution of (13) into (24) and integrating the GDD according to (17) finally leads to

$$
\begin{aligned}
m\left(k_{B}\right)= & \frac{1}{2 \pi} \sum_{v=0}^{\infty} \sum_{\mu=0}^{v}\left\{\frac{1}{v !(\mu+2)}\left(\begin{array}{c}
v \\
\mu
\end{array}\right) D_{(v+2)} c^{v+2}\left(-k_{0}\right)^{v-\mu}\right. \\
& \left.\cdot\left(1-\frac{\left|\kappa_{0}\right|}{\pi}\right)^{\mu+1} \cdot\left(\left(\kappa_{\mathrm{B}}^{\max }\right)^{\mu+2}-k_{\mathrm{B}}^{\mu+2}\right)\right\} \cdot
\end{aligned}
$$

Once this expression has been obtained and plotted, the inversion can always be obtained by inspection. In Section $\mathrm{V}-\mathrm{B}$, we discuss the complete design of a DCM starting from the analytical equation (26). This will show the usefulness of this approach for the analytical determination of an initial design that is already very close to the desired dispersion characteristic.

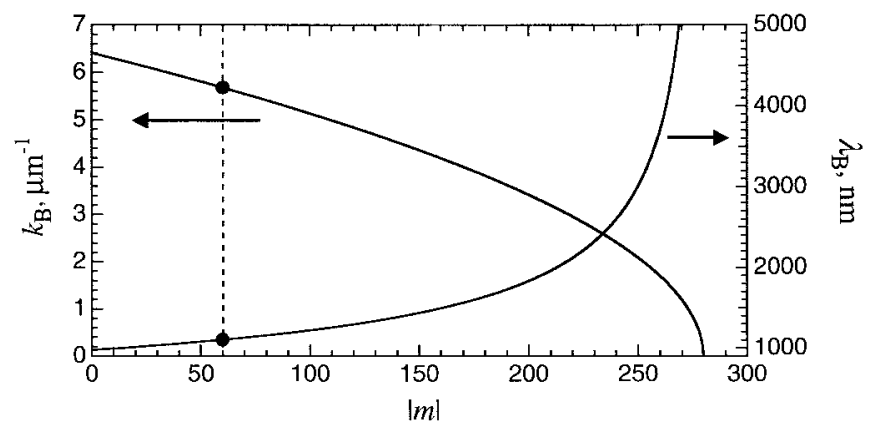

Fig. 4. Chirp law, which gives the Bragg wavenumber or Bragg wavelength as a function of the normalized position $|m|$ in the mirror, for a highly dispersive DCSM. In the case of a desired constant GDD, the chirp law with respect to Bragg wavenumber is a square root function according to (22). For the example discussed in the text, the chirp law has been used only over the first 60 index steps.

\section{NUMERICAL EXAMPLES}

\section{A. Double-Chirped Semiconductor Mirror}

Here, we discuss the design of a double-chirped semiconductor mirror (DCSM) with a constant negative GDD. This example illustrates the method and demonstrates the applicability of the theory not only to the design of chirped dielectric mirrors but also to the design of chirped Bragg gratings in general. However, we do not discuss the problem of matching the DCSM to air and the improvement of the design by computer optimization. A complete design including these issues is described in Section V-B.

Here, we use the constant refractive indices $n_{1}=3.0$ and $n_{\mathbf{h}}=3.5$, which are close to the refractive indices of AlAs and $\mathrm{GaAs}$, respectively, at around $1050 \mathrm{~nm}$. For these indices, the approximate coupling coefficient is $\kappa_{0} \approx-0.154$, according to (6). We desire a constant GDD. Thus, (22) is used as the chirp law for the Bragg wavenumber. For the desired GDD, a typical absolute value of $D_{0}=1000 \mathrm{fs}^{2}$ is assumed. A dispersion value at this order of magnitude is desired in many applications where large amounts of dispersion have to be compensated for within a single bounce on the mirror. The region of high reflectance and constant dispersion should be centered at around $1050 \mathrm{~nm}$. The value for the minimum Bragg wavelength is set to $\lambda_{\mathrm{B}}^{\min }=980 \mathrm{~nm}$, which corresponds to a maximum Bragg wavenumber of $k_{\mathrm{B}}^{\max }=2 \pi / 980 \mathrm{~nm} \approx$ $6.41 \mu \mathrm{m}^{-1}$.

Fig. 4 shows the Bragg wavenumber and Bragg wavelength with respect to the normalized distance in the mirror, obtained for the values above. Obviously, the Bragg wavenumber decreases to zero at $|m| \approx 280$. Thus, with the chirp law used here, one can only chirp the Bragg wavenumber over a

$$
T_{\mathrm{g}}(k)= \begin{cases}\frac{c D_{0}}{(\gamma+1) k_{0}^{\gamma}} \cdot\left\{\left(1-\frac{\left|\kappa_{0}\right|}{\pi}\right)^{\gamma+1}\left(k_{\mathrm{B}}^{\max }\right)^{\gamma+1}-k^{\gamma+1}\right\}, & \gamma \neq-1 \\ c D_{0} k_{0} \cdot \ln \left\{\left(1-\frac{\left|\kappa_{0}\right|}{\pi}\right) \frac{k_{\mathrm{B}}^{\max }}{k}\right\}, & \gamma=-1\end{cases}
$$




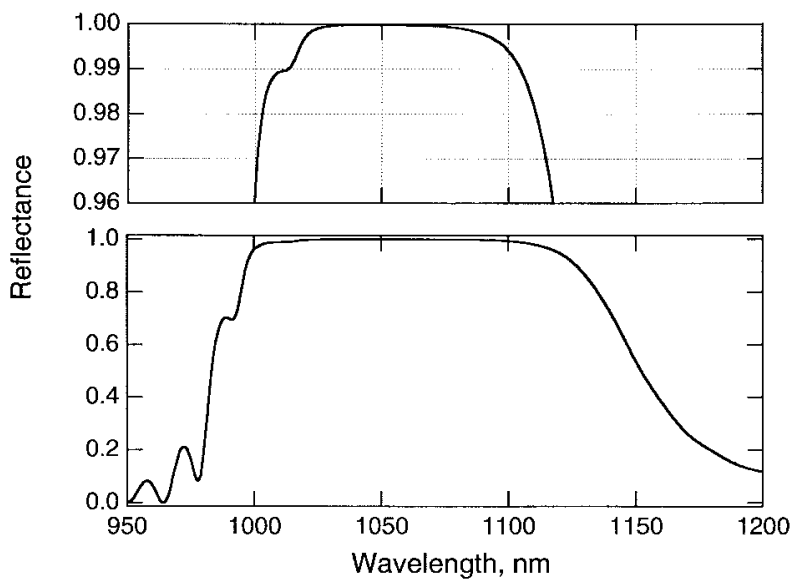

(a)
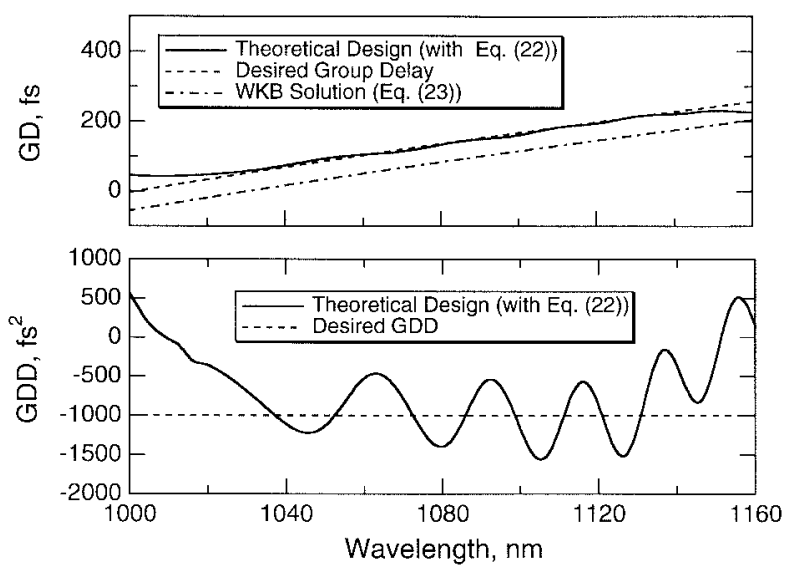

(b)

Fig. 5. Reflectance, group delay, and GDD of a double-chirped semiconductor Bragg mirror using the chirp law shown in Fig. 4. The thickness of the high-index layer was chirped as described in the text. (a) The bandwidth of the highly reflective region is about $100 \mathrm{~nm}$. (b) The designed GDD (solid line) oscillates around the desired constant GDD (dashed line). The difference between the designed group delay (solid line) and the group delay obtained from the WKB solution (dash-dotted line) is mostly due to the assumed constant coupling coefficient.

maximum distance of about 280 index steps. However, due to practical limitations arising from semiconductor growth (e.g., with MOCVD), the number of index steps cannot be much larger than 100. Thus, for a demonstration, we use 60 index steps. For the computation of the mirror properties, the Bragg wavenumber has to be taken at 60 discrete points defined by $|m|-0.5$ with $|m|=1,2, \cdots, 60$. The reflectance and phase properties upon reflection are exactly calculated with the transfer matrix formalism.

Fig. 5 shows the mirror reflectance, group delay, and GDD, where all properties are calculated without considering the index jump to an ambient medium. In order to avoid undesired oscillations, the impedance was matched very slowly over the first 50 index steps by an appropriate slow tapering of the coupling coefficient [7], [8]. Thus, the design presented here consists of only two parts, the double-chirp section (50 index steps) and a simple-chirp section (10 index steps). Here, the coupling coefficient was increased almost linearly via the thickness of the high-index layers according to $d_{\mathbf{h}, m} \pi /\left(2 k_{\mathrm{B}}(50) n_{\mathbf{h}}\right)(|m| / 50)^{1.05}$. The reason is that an
TABLE I

Center Wavelength and Dispersion Coefficients, According to (24), FOR the Desired GDD of a Dielectric DCM That CAN Be Used For DisPERSION COMPENSATION IN AN OPO, AS DESCRIBED IN THE TEXT

\begin{tabular}{c|c|c|c|c|c}
\hline$\lambda_{0}=\frac{2 \pi}{k_{0}},[\mathrm{~nm}]$ & $D_{(2)},\left[\mathrm{fs}^{2}\right]$ & $D_{(3)},\left[\mathrm{fs}^{3}\right]$ & $D_{(4)},\left[\mathrm{fs}^{4}\right]$ & $D_{(5)},\left[\mathrm{fs}^{5}\right]$ & $D_{(6)},\left[\mathrm{fs}^{6}\right]$ \\
\hline 615 & -44.7 & -21.0 & -2.3 & -6.0 & 3.5 \\
\hline
\end{tabular}

increase with a higher power law, e.g., with an exponent 2 , would lead to unrealistically thin high-index layers in the front part of the mirror. Additionally, a double chirp which is too strong would superimpose an additional dispersion effect that is undesired for the demonstration of the method described here.

As can be seen from Fig. 5(a), the high-reflectance range has a bandwidth of more than $100 \mathrm{~nm}$. However, due to the long matching section, a part of the high-reflectance range has been lost on the short wavelength side. This is why the minimum Bragg wavelength was chosen as short as $980 \mathrm{~nm}$. Fig. 5(b) shows the group delay and GDD. Obviously, the theoretical design is very close to the desired group delay and GDD, respectively. The designed GDD oscillates around the desired constant value. The average value is in excellent agreement with the desired value. The oscillations are due to imperfections in the double-chirp section and could be further reduced by methods such as numerical optimization. One has to note that the desired group delay is only determined up to a constant offset value. Therefore, we adjust the desired group delay for one wavelength. The dash-dotted line in Fig. 5(b) is the group delay obtained from the WKB solution (23) for the analytical chirp law (22) $(\gamma=0)$. The constant difference between the adjusted group delay and the WKB solution is about $50 \mathrm{fs}$. This difference is explained by the assumption of a constant coupling coefficient, according to (6). As we discussed in [8], that approximation leads to an almost constant difference between the exact computed group delay and the WKB solution. However, this is irrelevant for the physically most important quantity, which is the GDD.

\section{B. Design of a Double-Chirped Dielectric Mirror}

Here, we discuss the complete design of a dielectric DCM with a desired dispersion characteristic, given as a Taylor expansion (24) up to sixth order. The center wavelength and dispersion coefficients are summarized in Table I. Six bounces on this mirror should compensate for the material dispersion introduced by a 2-mm-long BBO crystal and $1 \mathrm{~mm}$ of fused silica [12]. It is of considerable interest to have broadband dispersion compensating mirrors at around $600 \mathrm{~nm}$ for short-pulse OPO's [16], [17] and optical parametric amplifiers (OPA's) [18], [19] in that wavelength range.

For the numerical evaluation of (26), the maximum Bragg wavenumber was chosen to be $k_{\mathrm{B}}^{\max }=2 \pi / 500 \mathrm{~nm} \approx$ $12.57 \mu \mathrm{m}^{-1}$. For the coupling coefficient, we used the constant value $\kappa_{0}=-0.481$, which is obtained from (6) for the indices $n_{1}=1.50$ and $n_{\mathbf{h}}=2.45$. These indices are close to the indices of $\mathrm{SiO}_{2}$ and $\mathrm{TiO}_{2}$ at wavelengths around $\lambda=600 \mathrm{~nm}$. 


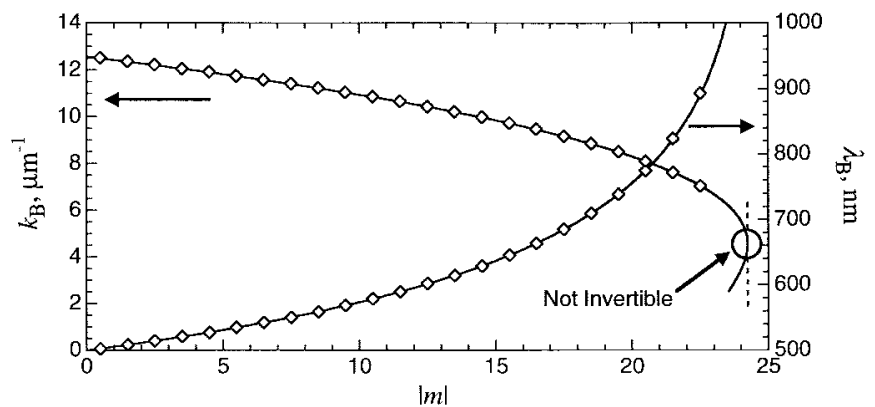

Fig. 6. Chirp law for a dielectric DCM with a desired GDD, as summarized in Table I. The minimum Bragg wavelength was chosen to be $500 \mathrm{~nm}$. At $|m| \approx 24$, the inverse chirp law is not invertible due to the increase of the desired GDD for longer wavelengths [see Fig. 7(b)]. Also shown are the 23 discrete points at which the chirp law was evaluated in order to generate the theoretical designs shown in Fig. 7. The dashed vertical line symbolizes the position in the mirror where all wavelengths around the Bragg wavelength are reflected, when a quarter-wave section follows after the simple-chirp section.

Fig. 6 shows the Bragg wavenumber and Bragg wavelength again as a function of the position in the mirror. One can see that, for this example, (26) is not a one-to-one function. This means that, at $|m| \approx 24$, the inverse chirp law (24) is locally not invertible. The occurrence of this phenomenon is discussed in detail in Section V-C. Thus, using this law, one can chirp the Bragg wavenumber only over a maximum number of 24 index steps. In order to generate a mirror design with not too many layers, the design presented here was chirped over 23 index steps. The 23 discrete points for the Bragg wavenumbers, where the chirp law is evaluated, are also shown in Fig. 6.

Fig. 7 shows the mirror properties for a final design that can be fabricated. This design is achieved by starting from the analytical design obtained with the discrete points for the Bragg wavenumber from Fig. 6. Additionally, the problem of matching to air is solved by putting a broad-band AR coating on top of the mirror [7], [8]. Finally, the design performance is improved by computer optimization with a standard gradient algorithm from [20]. It is important to note that, for the refinement procedure and calculation of the final mirror properties, the wavelength dependence of the refractive indices as well as the absorption and scattering losses have to be taken into account. In contrast, this is not necessary for the determination of the discrete Bragg wavenumbers from (26), because these effects are considered to be small. For the generation of the analytical initial design, the thickness of the high-index layer was almost linearly chirped over the first 10 index steps according to $d_{\mathbf{h}, m}=\pi /\left(2 k_{\mathrm{B}}(10) n_{\mathbf{h}}\right)(|m| / 10)^{1.05}$.

Fig. 7(a) shows the reflectance and group delay of the final design. The reflectance is high over a bandwidth of $250 \mathrm{~nm}$ $(500-750 \mathrm{~nm})$, and the group delay is very smooth over a bandwidth of about $180 \mathrm{~nm}(540-720 \mathrm{~nm})$. In this range, the deviation from the desired group delay is only $0.14 \mathrm{fs}$ (rms). As is demonstrated by Fig. 7(b), the designed GDD oscillates around the desired GDD in this region, with an oscillation amplitude of $\approx 6-7 \mathrm{fs}^{2}$. Although the starting design is computer optimized, the oscillations in the GDD cannot be removed completely. The reason is that the AR coating does not allow the generation of a perfect matching to the ambient medium (air) over a bandwidth of $180 \mathrm{~nm}$.

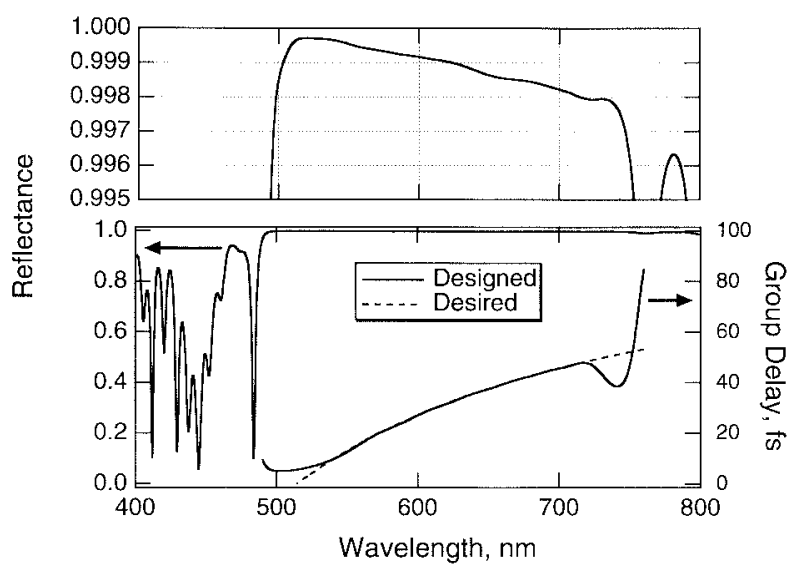

(a)
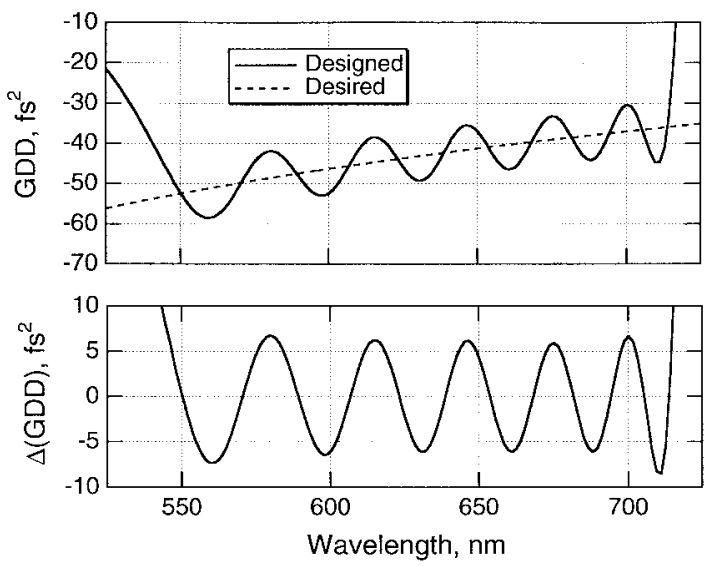

(b)

Fig. 7. Broad-band design of a DCM that is useful for dispersion compensation in an OPO. The designed reflectance, group delay, and GDD are obtained using the chirp law shown in Fig. 6. The thickness of the high-index layer was chirped as described in the text. The mirror properties are shown after adding an AR coating on top of the mirror structure and a subsequent computer refinement. (a) The mirror is highly reflective over a bandwidth of about $250 \mathrm{~nm}$. The designed group delay (solid line) is extremely smooth and is very close to the desired group delay (dashed line) over a bandwidth of about $180 \mathrm{~nm}$. (b) The designed GDD (solid line, upper plot) oscillates around the desired GDD (dashed line, upper plot) with an amplitude of about 6-7 $\mathrm{fs}^{2}$ in that range. The lower plot shows the difference between the designed and desired GDD.

Fig. 8 shows the physical layer thicknesses of the starting design and of the final design after computer optimization. The total design consists of 54 layers, in which 46 layers have been used for the theoretical DCM structure and 8 layers for the AR coating. The layer thicknesses of the AR coating are only shown after optimization. The reason is that we started from a 12-layer AR coating, but during the optimization procedure it was possible to eliminate the two thinnest layers of the AR coating leading to an effective reduction of 4 layers. The figure illustrates two facts. The first observation is that the analytical initial design of the DCM does not change very much during optimization. Most corrections to the starting design occur in the AR coating in the front part of the total mirror. The other observation is that the different sections of the DCM can still be clearly identified. In the AR coating, the layer thicknesses seem to change randomly, whereas the double-chirp section starts with very thin high- 


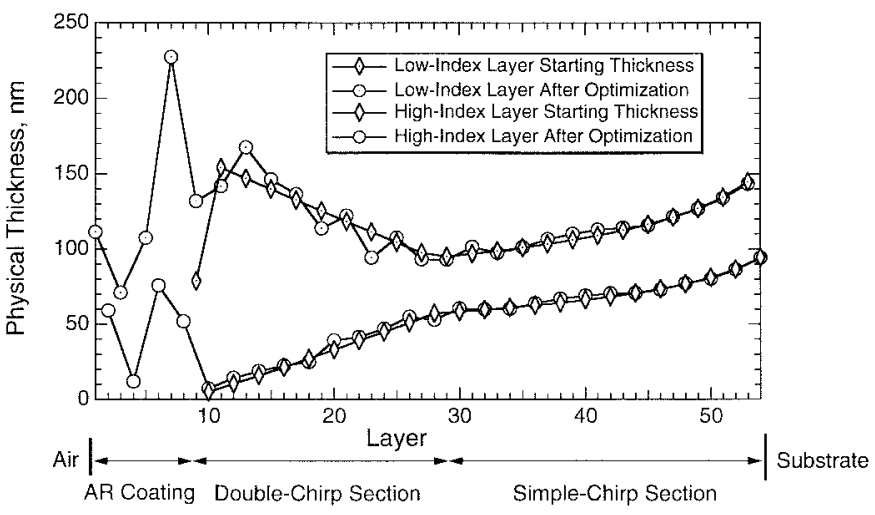

Fig. 8. Layer thicknesses of the DCM design corresponding to the mirror properties shown in Fig. 7. The thicknesses of the high- and low-index layers are shown before and after computer optimization. The layer thicknesses of the AR coating are only shown after optimization because the two thinnest layers of the AR coating are removed during the refinement procedure. Obviously, the different sections of a DCM can still be clearly identified even after computer optimization.

index layers with increasing thicknesses along the DCM. The thickness of the low-index layers decreases in the doublechirp section. In the simple-chirp section, the thicknesses of the high- and low-index layers increase simultaneously. The stability of the starting design against changes due to the optimization procedure is understood by the fact that a sufficiently good AR coating can be designed over a bandwidth of about $180 \mathrm{~nm}$ at wavelengths around $600 \mathrm{~nm}$. If one tries to extend the bandwidth with smooth dispersion properties beyond the bandwidth of the AR coating, the optimization will significantly change the layer thicknesses of the DCM structure. Additionally, the amplitude of the oscillation in the GDD will increase with the required bandwidth.

The mirror design presented here will be manufactured in the near future. If the properties of the fabricated mirrors will be close to the designed properties, ultrashort laser pulses from an OPO with a duration of about 5-7 fs can be expected [12]

\section{Unfavorable Choice of the Desired Dispersion Characteristic}

The analytical discussion of the dispersion behavior of DCM's gives insight into reasons why certain dispersion characteristics might be difficult, if not impossible, to achieve. For example, such a situation arises if the absolute value of the desired second-order dispersion coefficient $D_{(2)}$ is chosen too low or too high, or if the ratio between the different dispersion coefficients $D_{(v+2)}$ is chosen in an unfavorable way. Generally, one has to chirp relatively slowly to obtain a high amount of negative dispersion and relatively quickly for a small amount. As a consequence, in the first case, one needs many index steps to achieve a broad enough high-reflectance bandwidth. In the second case, it is possible that the reflectance is reduced due to the fast chirp over a small number of index steps. Thus, for given materials, there is always an optimum range for the dispersion, over which a high reflectance and smooth GDD can be achieved simultaneously.

The great advantage of the design method described in this paper is that situations, in which the optimum range is left, are clearly indicated by plotting and analyzing the chirp law, derived from (21) or (26). As an example, the noninvertible region in Fig. 6 at a finite Bragg wavelength is a consequence of the increase of the desired GDD with respect to wavelength [see Fig. 7(b)]. In fact, the critical Bragg wavenumber at which this happens corresponds exactly to the wavenumber where the desired GDD becomes positive. Using the values for the design discussed in Section V-B, the critical Bragg wavenumber is $k_{\mathrm{B}}^{\text {crit }} \approx 4.56 \mu \mathrm{m}^{-1}\left(\lambda_{\mathrm{B}}^{\text {crit }} \approx 1378 \mathrm{~nm}\right)$ and the wavenumber for vanishing dispersion is found to be $k^{\text {crit }} \approx 3.86 \mu \mathrm{m}^{-1}\left(\lambda^{\text {crit }} \approx 1628 \mathrm{~nm}\right)$. These wavenumbers are connected via (13). Now, it is quite easy to understand what happens. As long as the desired GDD is negative, the Bragg wavenumber is a monotonically decreasing function. When the desired GDD becomes positive, the direction of the chirp has to be changed, which means that the Bragg wavenumber should now increase. Of course, the formulas derived from WKB solutions cannot cover this case. Additionally, the fast chirp for the longer wavelengths also explains the problem of keeping the reflectance at a high level, as is demonstrated by the decrease of the reflectance with respect to wavelength [see Fig. 7(a)]).

A quarter-wave section following the simple-chirp section offers a possible solution over a limited wavelength range when the noninvertible region is reached. The quarter-wave stack solves both problems: it increases the reflectance and leads to an almost vanishing dispersion for wavelengths around the corresponding Bragg wavelength. Thus, in such a case, the Bragg mirror can be considered as the "natural" continuation of the simple-chirp section, symbolized by the dashed vertical line in Fig. 6. The Bragg wavelength of the quarter-wave section is a free parameter which can be adjusted to achieve maximum performance. It should be chosen much smaller than $\lambda_{\mathrm{B}}^{\text {crit }}$ and close to the last value used in the simple-chirp section. However, this method works only over a restricted wavelength range.

The conclusion is that, if the inverse chirp law is not invertible in the region of interest, as described above, the design method proposed in this paper will not lead to an appropriate theoretical starting design for later computer refinement. A possible solution is to change the desired dispersion characteristic or to add a quarter-wave stack after the simplechirp section. On the other hand, the failure of producing a reasonable initial design indicates that an optimum design for a high-reflectance coating with the desired dispersion might not exist.

\section{CONCLUSION}

We have presented an analytical design method for DCM's or general chirped Bragg gratings with a given dispersion characteristic. Problems in the mirror design that may arise due to an unfavorable choice of the desired GDD are indicated by the corresponding chirp law. The chirp law also directly indicates how many layers have to be used for the theoretical design in order to achieve the desired dispersion characteristic over a given bandwidth. The applicability of our design method has been demonstrated by two examples, a semiconductor and a 
dielectric DCM. As has been shown, the analytical designs achieved by this method are very close to the desired design goals and can be used as excellent starting designs for a subsequent computer optimization.

\section{REFERENCES}

[1] J. Zhou, G. Taft, C.-P. Huang, M. M. Murnane, and H. C. Kapteyn, "Pulse evolution in a broad-bandwidth Ti:sapphire laser," Opt. Lett., vol. 19, pp. 1149-1151, 1994.

[2] A. Stingl, M. Lenzner, Ch. Spielmann, F. Krausz, and R. Szipöcs, "Sub10 fs mirror-dispersion-controlled Ti:sapphire laser," Opt. Lett., vol. 20 , pp. 602-604, 1995.

[3] I. D. Jung, F. X. Kärtner, N. Matuschek, D. H. Sutter, F. Morier-Genoud, G. Zhang, U. Keller, V. Scheuer, M. Tilsch, and T. Tschudi, "Selfstarting 6.5 fs pulses from a Ti:sapphire laser," Opt. Lett., vol. 22, pp. 1009-1011, 1997.

[4] D. H. Sutter, I. D. Jung, F. X. Kärtner, N. Matuschek, F. Morier-Genoud, V. Scheuer, M. Tilsch, T. Tschudi, and U. Keller, "Self-starting 6.5-fs pulses from a Ti:sapphire laser using a semiconductor saturable absorber and double-chirped mirrors," IEEE J. Select. Topics Quantum Electron., vol. 4, pp. 169-178, 1998.

[5] R. Szipöcs, K. Ferencz, Ch. Spielmann, and F. Krausz, "Chirped multilayer coatings for broadband dispersion control in femtosecond lasers," Opt. Lett., vol. 19, pp. 201-203, 1994.

[6] A. V. Tikhonravov, P. W. Baumeister, and K. V. Popov, "Phase properties of multilayer coatings," Appl. Opt., vol. 36, pp. 4382-4392, 1997

[7] F. X. Kärtner, N. Matuschek, T. Schibli, U. Keller, H. Haus, C. Heine, R. Morf, V. Scheuer, M. Tilsch, and T. Tschudi, "Design and fabrication of double-chirped mirrors," Opt. Lett., vol. 22, pp. 831-833, 1997.

[8] N. Matuschek, F. X. Kärtner, and U. Keller, "Theory of double-chirped mirrors," IEEE J. Select. Topics Quantum Electron., vol. 4, pp. 197-208, 1998.

[9] ings with arbitrary strong index modulations," IEEE J. Quantum Electron., vol. 33, pp. 295-302, 1997.

[10] L. Poladian, "Graphical and WKB analysis of nonuniform Bragg gratings," Phys. Rev. E, vol. 48, pp. 4758-4767, 1993.

[11] J. E. Sipe, L. Poladian, and C. M. de Sterke, "Propagation through nonuniform grating structures," J. Opt. Soc. Amer. A, vol. 11, pp. 1307-1320, 1994.

[12] G. M. Gale, private communication 1998

[13] J. A. Dobrowolski, A. V. Tikhonravov, M. K. Trubetskov, B. T. Sullivan, and P. G. Verly, "Optimal single-band normal-incidence antireflection coatings," Appl. Opt., vol. 35, pp. 644-658, 1996.

[14] H. A. Haus, Waves and Fields in Optoelectronics. Englewood Cliffs, NJ: Prentice-Hall, 1998.

[15] N. Matuschek, F. X. Kärtner, and U. Keller, "Double-chirped mirror designs with adjustable higher order dispersion," in Proc. Conf. Lasers and Electrooptics (CLEO '98), San Francisco, CA, May 3-8, 1998, paper CThC6.

[16] G. M. Gale, M. Cavallari, and F. Hache, "Femtosecond visible optical parametric oscillator," J. Opt. Soc. Amer. B, vol. 15, pp. 702-714, 1998.

[17] G. M. Gale, F. Hache, and M. Cavallari, "Broad-bandwidth parametric amplification in the visible: Femtosecond experiments and simulations," IEEE J. Select. Topics Quantum Electron., vol. 4, pp. 224-229, 1998.

[18] G. Cerullo, M. Nisoli, and S. De Silvestri, "Generation of 11 fs pulses tunable across the visible by optical parametric amplification," Appl. Phys. Lett., vol. 71, pp. 3616-3618, 1997.

[19] A. Shirakawa, I. Sakane, and T. Kobayashi, "Pulse-front-matched optical parametric amplification for pulse generation tunable in the visible and near-infrared reaching to $6.5 \mathrm{fs}$," in Proc. Conf. Lasers and Electrooptics (CLEO '98), San Francisco, CA, May 3-8, 1998, paper CPD11-2.

[20] W. H. Press, S. V. Teukolsky, W. T. Vetterling, and B. P. Flannery, Numerical Recipes in Fortran, 2nd ed. Cambridge, U.K.: Cambridge Univ., 1994.

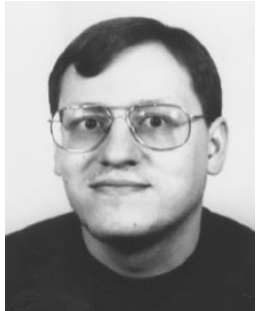

Nicolai Matuschek was born in Biberach an der Riß, Germany, in 1968. He received the diploma degree in physics from the University of Ulm, Ulm, Germany in 1995. He is currently working toward the Ph.D. degree at the Institute of Quantum Electronics, Swiss Federal Institute of Technology (ETH), Zurich, Switzerland

After finishing his diploma degree in the field of theoretical polymer physics, he changed his research interests to laser physics, where he is currently focused on the theory of mode-locked solid-state lasers.

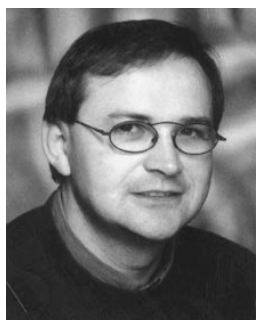

Franz X. Kärtner was born in Cham, Germany, in 1961. He received the diploma degree in electrical engineering from the Technical University Munich, Munich, Germany, in 1986 and the Ph.D. degree for work on noise in microwave circuits and optical devices in 1989

From 1991 to 1993, he was a Feodor-Lynen Research Fellow of the Humboldt Foundation at Massachusetts Institute of Technology (MIT), Cambridge, MA, where he carried out research on the generation of squeezed states in fibers and quantum nondemolition measurements. From 1993 to 1997, he was with the Institute of Quantum Electronics, Swiss Federal Institute of Technology (ETH), Zurich, Switzerland. He received his Habilitation in 1997 with work on ultrashort pulse generation. Since 1998, he has been a Visiting Assistant Professor at MIT.

Dr. Kärtner is a member of the German Physical Society, the Optical Society of America, and the IEEE.

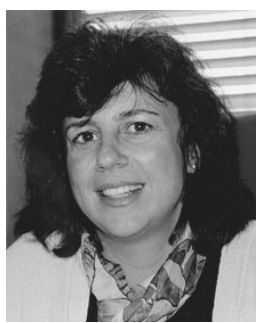

Ursula Keller (M'89) was born in Zug, Switzerland, in June 1959. She received the "Diplom" in physics from the Swiss Federal Institute of Technology (ETH), Zurich, Switzerland, in 1984 and the M.S. and Ph.D. degrees in applied physics from Stanford University, Stanford, CA, in 1987 and 1989, respectively. Her Ph.D. research was in optical probing of charge and voltage in GaAs integrated circuits and in low-noise ultrafast laser systems.

From late 1984 to 1985 , she worked on optical bistability at Heriot-Watt University, Edinburgh, Scotland, U.K. In 1989, she joined AT\&T Bell Laboratories, Holmdel, NJ, as a Member of Technical Staff, where she conducted research on photonic switching, ultrafast laser systems, and semiconductor spectroscopy. Since March 1993, she has been an Associate Professor and since October 1997 a Full Professor in the Physics Department at the ETH, Zurich. Her current research interests are in ultrafast lasers, spectroscopy, local probes, and novel devices for applications in optical information processing, communication and medicine. She has published more than 80 journal papers and one book chapter and holds five patents.

Prof. Keller is a member of the Optical Society of America, the European Physical Society, the Swiss Physical Society, and the Swiss Academy of Technical Sciences. During 1985-1986, she was a Fulbright Fellow, in 1987-1988 she received an IBM Predoctoral Fellowship, and in 1998 she received the Carl Zeiss Research Award. 